\title{
The Influence of Traits Associated with Autism Spectrum Disorder (ASD) on the Detection of Fake News
}

\author{
Jacqui Taylor-Jackson \& Sophie Matthews
}

\begin{abstract}
A small-scale exploratory study was conducted to investigate whether students with traits associated with Autism Spectrum Disorder (ASD) were more or less accurate in spotting different types of fake news. A non-clinical sample of university students completed an online identification task, where both fake and real articles items were manipulated in terms of their emotive content. When individuals with low and high scores on the Autism-Spectrum Quotient (Baron-Cohen et al., 2001) were compared, there were no significant main effects on detection accuracy. However, there were two significant interactions, indicating an interesting relationship between message emotiveness, ASD and fake news detection. The results contribute to an understanding of how individual differences, in particular ASD, affect judgements of news and will contribute to tailoring education regarding features of fake news.
\end{abstract}

\section{Literature Review}

\section{Background}

Fake news has been defined as, "false stories that appear to be news, spread on the internet or using other media, usually created to influence political views or as a joke" (Cambridge Advanced Learner's Dictionary, 2018). Other definitions focus on the intentional fabrication of news to mimic real news content, to mislead others, (Lazer, Baum, Benkler, Berinsky, Greenhill, Menczer \& Schudson, 2018). The term has grown in popularity and use, particularly so during and after the 2016 American election (Allcott \& Gentzkow, 2017). Much of the previous research has focussed on factors that affect the believability of fake news. For example, Pennycook, Cannon \& Rand (2017) found that when fake news headlines gathered from social media were displayed, just a single prior exposure to the item increased the perception that the headline was perceived as accurate (a 'familiarity effect'). Believability was further increased when the headline was labelled as 'contested by fact checkers' or supported by a perceived reliable source.

\section{$\underline{\text { Individual psychological characteristics and fake news }}$}

The implications of spreading fake news can be great and far-reaching, yet there is relatively little research investigating factors relating to individual psychological characteristics and detection of fake news. Pennycook $\&$ Rand (2019) investigated the 'cognitive psychological profile' of individuals and their ability to differentiate fake news from real news. They found that those individuals with a less analytical thinking style and those who had a tendency to believe information based on its conformity with their own ideology were poorer at differentiating fake news. Pennycook and Rand (2019) explain this by saying that people may believe in fake news because they 'fail to think'. This is supported by the finding that through analytical and open-minded thinking, people can help to protect themselves from disinformation (Bronstein, Pennycook, Bear, Rand \& Cannon, 2019).

\section{$\underline{\text { Autism Spectrum Disorder }}$}

Individuals with traits associated with Autism Spectrum Disorder (ASD) understand information in different ways compared with those individuals without these traits and they may perceive emotion in different ways. ASD as outlined in the ICD-11 (2018), is a lifelong neuro-developmental disorder that is believed to affect $1 \%$ of the population, at a male to female ratio of approximately 2 to 1 . ASD can cause an individual to experience difficulties in social and communication aspects of everyday life that are severe enough to result in impairments in other aspects of functioning (ICD-11, 2018; Baron-Cohen et al., 2009; Carpenter, Happé \& Egerton, 2019). Individuals with ASD may display inflexible patterns of behaviour, deficits in Theory of Mind, (recognising the mental states of others), and / or deficits in their ability to empathise with others (Baron-Cohen, Leslie \& Frith, 1985; Smith, 2009). As ASD is a spectrum condition, while many individuals with the condition may share 
symptoms, each individual with ASD will have their own unique set of symptoms and difficulties. However, despite research focussing on 'difficulties', other research has focussed on the positive abilities of individuals displaying ASD traits, for example, Hayashi, Kato, Igarashi \& Kashima (2008) found that individuals with ASD exhibit superior fluid intelligence (the ability to solve novel problems and reason independently from previous knowledge), when compared to a control group.

Brosnan, Chapman and Ashwin (2014) reported that individuals with ASD showed a more circumspect decision-making style, (rather than jumping to conclusions) and DeMartino et al. (2008) suggest that there is a level of reliance upon rational and logical decision making for individuals with ASD. In a study investigating the life experiences of individuals with high functioning ASD, Luke, Clare, Ring, Redley \& Watson (2012) found that these individuals find decision making to be exhausting and may often avoid decision making if they can. Bronsan, Chapman and Ashwin (2014) also propose that an association between decision-making and anxiety or mental 'freezing' can result in ASD individuals delaying the decision-making process. Pennycook \& Rand (2019) propose that susceptibility to fake news can be explained by lack of reasoning, therefore this might suggest that individuals with ASD traits would be less susceptible.

\section{$\underline{\text { Emotiveness and fake news }}$}

Fake news is often created to include highly affective content, particularly through the use of images and other visual cues (Bakir \& McStay, 2018). Affective content is used to provoke interest, and to arouse emotion and empathy in the reader (Grabe, Zhou \& Barnett, 2001), while at the same time often forsaking the accuracy of the information presented (Molek-Kozakowska, 2013). DeMartino et al. (2008) found that individuals with autism pay less attention to emotional context when making decisions. Therefore, due to differences in the way that emotions are interpreted, those individuals high in autistic traits may be less affected by emotive content in fake news, compared to those with less traits. Horne and Adah, (2017), suggest that when attempting to differentiate between fake and real news, the title or headline of an article can be a strong indicator, due purely to its wording and structure. Headlines using emotive language are often associated with topics such as scandal, crime or disaster (Chesney, Liakata, Poesio \& Purver, 2017). Exaggeration, embellishment and use of emotive language is often a prominent feature in sensationalist news, especially fake news and clickbait headlines (MolekKozakowska, 2013).

\section{Hypotheses}

Two hypotheses are proposed:

H1: Individuals with higher levels of autistic traits will correctly identify more articles as being real or fake, compared to individuals with low levels of autistic traits

$\mathrm{H} 2$ : When articles are emotive, individuals with higher levels of autistic traits will correctly identify more articles as being real or fake, compared to those with low levels of autistic traits.

\section{Method}

\section{Design}

The study used a 2x2x2 mixed factorial design. Two between-subjects independent variables were: whether the article was emotive or not emotive, and whether the article was real or fake. The within-subjects independent variable categorised participants as having high or low levels of autistic traits (measured using the AutismSpectrum Quotient by Baron-Cohen, Wheelwright, Skinner, Martin \& Clubley, 2001). This categorisation was based on a median split of the data, where participants scoring 15 or higher on the Autism-Spectrum Quotient were assigned to the high-level group, those scoring less than 15 to the low-level group.

\section{Participants}

Participants consisted of a volunteer sample of 35 students. The sample had a mean age of 20.69 years and was made up of 3 male and 32 female participants. A separate sample, used to pilot materials, consisted of 4 participants ( 1 male and 3 females), with a mean age of 22 years. All participants were rewarded with 0.5 course participation credits.

\section{Materials}

The Autism-Spectrum Quotient (Baron-Cohen, Wheelwright, Skinner, Martin \& Clubley, 2001) was designed to measure autistic traits in adults of normal intelligence, and to not be significantly affected by IQ. The Autis mSpectrum Quotient was chosen as it is a measure of autistic traits that is not used for clinical assessment or diagnosis of ASD. However, it is effective at measuring traits associated with the disorder and it has been used in many studies involving non-clinical populations. Baron-Cohen et al. provide evidence that the questionnaire 
has face and construct validity and that it is a valid measure of the five main points of interest that are considered in ASD; social, communication, imagination, attention to detail and attention switching. Twenty article headlines with an accompanying image were developed: ten of these were sourced from real news reports, whilst the remaining were 'fake news', created for this study. The articles were piloted to ensure that the articles were of similar levels of difficulty for participants to determine whether they were real or fake. Both fake and real articles were initially categorised by one of the co-authors as either emotive or not emotive, with an equal number of fake and real articles in each category. In order to categorise these articles, the definition of 'emotive' as given by the Oxford Dictionary of English (Stevenson, 2010) was used, where an emotive issue is one 'expressing a person's feelings rather than being neutrally descriptive'. As suggested by Chesney, Liakata, Poesio \& Purver (2017), use of emotive language often relates to particular topics in fake news and evokes emotion or empathy from the reader. The pilot sample then also categorised the article headlines and two discrepancies were discussed and a final set of headlines was produced.

$\underline{\text { Procedure }}$

After participants gave their informed consent and their age and gender collected, they were asked to rate on a scale of 10 how confident they believed themselves to be at detecting fake news, giving an initial confidence score. After this, each article was displayed, and participants were asked to decide whether it was real or fake and give a confidence rating out of ten. Participants were also given the option to give reasons for their decision. Once all 20 articles had been displayed, participants completed the Autism-Spectrum Quotient. After completing the study, participants were thanked and debriefed and informed as to which articles were fake and the role of emotiveness.

\section{Results}

A $2 \times 2 \times 2$ mixed ANOVA statistical test was conducted. The manipulation of emotiveness was not significant, $F(1,33)=.90, p=.35$, therefore, whether the article was emotive or not made no significant difference upon participant's correct identification of fake and real articles. The main effect of level of autistic traits was also not significant, $F(1,33)=.98, p=.33$, suggesting that having high or low autistic traits does not affect an individual's ability to correctly identify fake and real news articles. The main effect of article type (fake or real) was significant, $F(1,33)=5.89, p=.02$. This suggests that whether the article presented was real or fake had a significant effect on participant's decision, and whether the article was identified correctly as real or fake.

There was a significant interaction between level of emotiveness and level of autistic traits, $F(1,33)=7.50, p=$ .01 . Figure 1 illustrates this interaction and shows that for individuals with a low level of autistic traits, the article emotiveness significantly affected detection rates; where the high emotive articles were correctly identified more that the non-emotive articles.

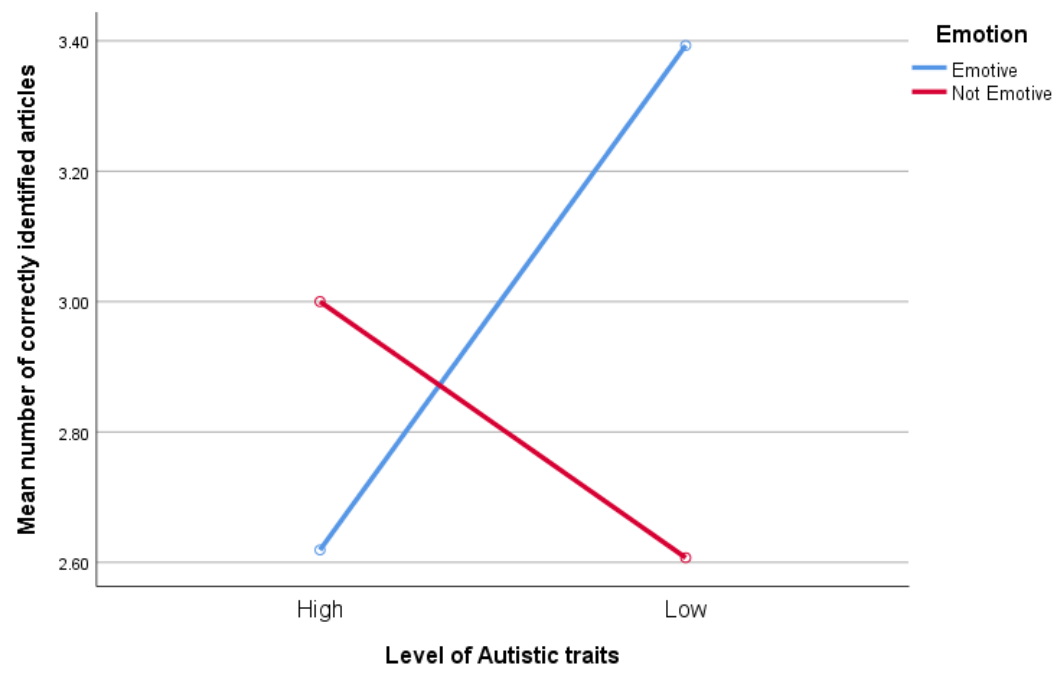

Fig 1. The interaction between emotiveness of articles (emotive or non-emotive) and level of autistic traits (low or high). 
The interaction between emotiveness and article type (fake or real) was also significant, $F(1,33)=13.38, p=$ $<.01$ (Fig. 2). This indicates that when articles were emotive, they were significantly more likely to be correctly identified when they were real, compared to when they were fake. The interaction between level of autistic traits and article type was not significant, $F(1,33)=.58, p=.63$ (as illustrated in Fig. 3).

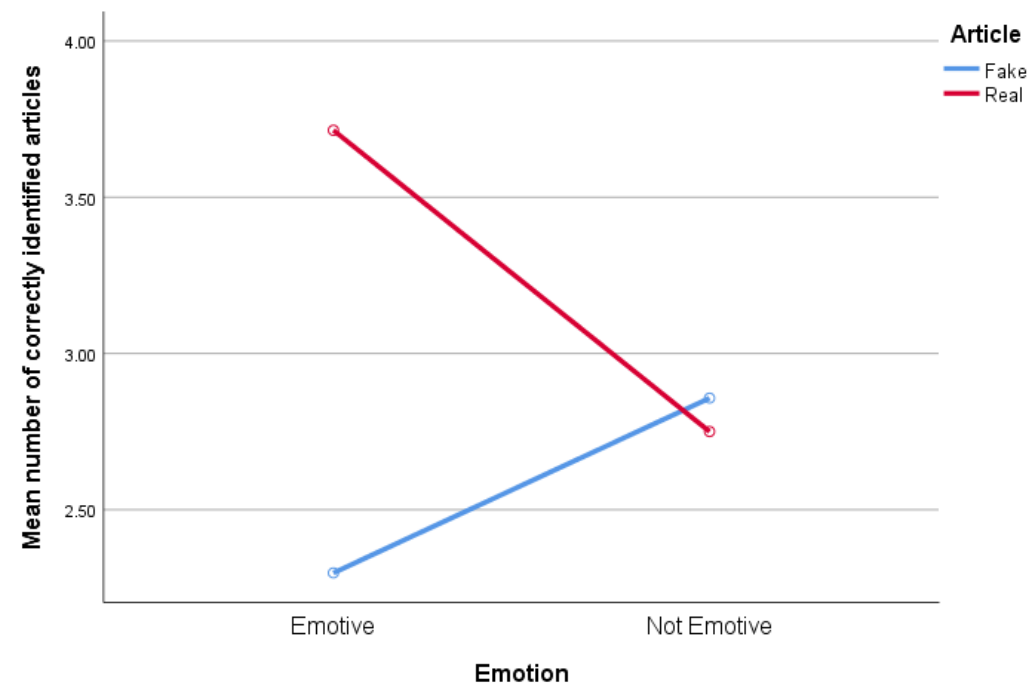

Fig. 2. The interaction between emotiveness of articles (emotive or non-emotive) and article type (real or fake).

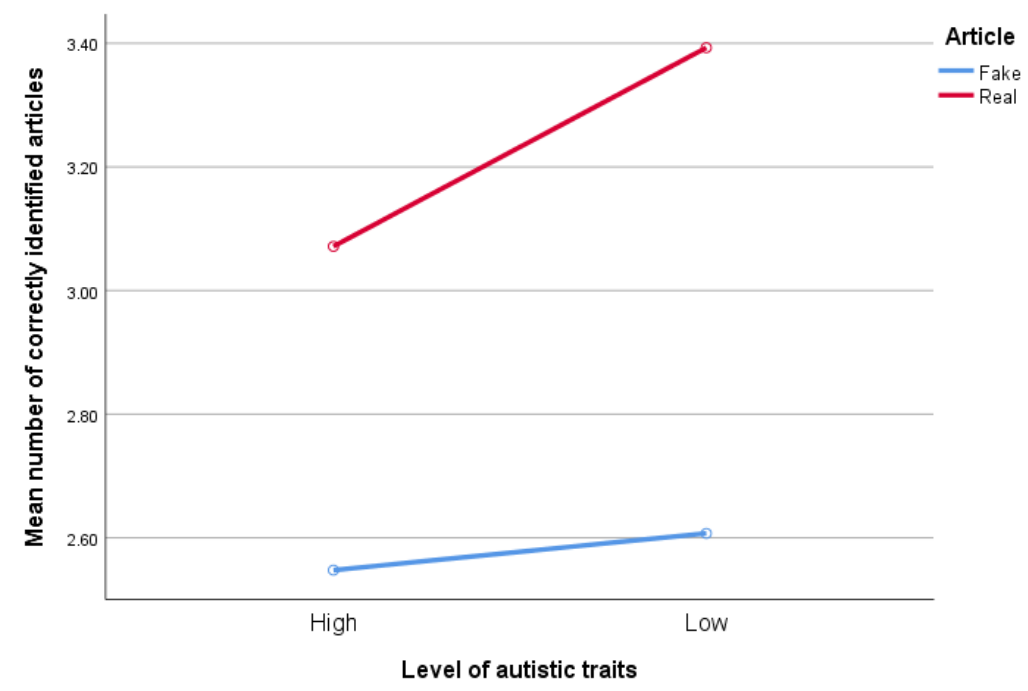

Fig. 3. The interaction between article type (real or fake) and level of autistic traits (low or high).

An independent-samples t-test compared individuals with high and low levels of autistic traits on their initial confidence scores. Although participants in the group with low levels of autistic traits rated their own fake news detection ability to be higher $(M=6.79, S D=1.53)$, compared to those in the group with high levels of autistic traits $(M=6.33, S D=1.85)$, this was not significant, $t(33)=.76, p=.45$, Cohen's $d=0.26$.

\section{Discussion}

This study found no significant main effect for level of autistic traits, suggesting that having high or low levels of autistic traits does not significantly affect the correct identification of 
fake and real news articles. Therefore, Hypothesis 1 that those who display higher levels of autistic traits will correctly identify more articles as being real or fake than those with low levels of autistic traits is rejected and the null hypothesis is accepted. Both groups correctly identified a higher number of real articles than fake news articles (illustrated in Fig. 3) and this was statistically significant. One potential reason for this may be due to the fact that the real news articles were sourced from real world news and therefore may have been seen previously by participants before they took part in the study. In future research, fake news articles should be sourced from real fake news and not manipulated to appear as fake news.

The results showed no significant main effect of emotiveness on correct identification of fake news articles, however two significant interactions between emotiveness and autistic traits and between emotiveness and article type were found. As shown in Figure 1, individuals with low levels of autistic traits correctly identified significantly more articles when they were emotive than when they were non-emotive. Therefore Hypothesis 2, that individuals with higher levels of autistic traits will be influenced by the level of emotiveness, is accepted. A significant interaction was also found between emotiveness and article type for all participants. Figure 2 shows that emotive content played a prominent role in participant's decision making. When articles were both emotive and real, performance almost doubled from that when articles were emotive and fake. While correct detection for the non-emotive articles was the same for fake and real articles. This provides support for the findings of Bakir \& McStay (2018), who suggest that fake news is intentionally created to include highly affective content; when fake news articles are more emotive, participants are less accurate at correctly identifying them as fake, compared to emotive real articles. This provides support for DeMartino, Harrison, Knafo, Bird \& Dolan, (2008) that individuals with ASD may be less affected by the affective or emotive content included in fake news and the wider research showing that those with ASD are less likely to take emotional context into account when making decisions. Future research is needed to further investigate how emotive or affective content influences decision making in those with ASD.

An independent samples t-test analysing the initial confidence ratings given by participants, regarding how good they believed themselves to be at detecting fake news, and autistic traits was not significant. Considering the difficulties and reported dislike of decision-making as highlighted in the literature, it may have been believed that perhaps those with ASD would rate their own ability as worse than those without ASD. Future research is needed to explore ASD and confidence in decision-making. Also, further research could collect the time needed to make decisions regarding the judgment of fake news accuracy considering that Goldstein, Johnson \& Minshew (2001) and Brosnan, Chapman and Ashwin (2014) found that individuals with ASD took longer to make decisions.

Future research could explore the reasons why people believe news to be real or fake, and what features of fake news articles participants use in order to identify them as fake. In contrast to the many quantitative studies, this could be done through an in-depth qualitative analysis of reasons individuals give for believing an article to be true or fake. Accounts could then be compared for individuals with high and low levels of autistic traits.

It is important to recognise that it is possible that none of the sample had clinically diagnosed ASD. Further research needs to use a sample of participants with clinically diagnosed ASD. Although a valid measure of traits that are associated with ASD, the Autism-Spectrum Quotient is not one used for clinical diagnosis. Another limitation that may have arisen from measuring only traits associated with ASD is that when performing a median split upon the 
data collected from participants, those who scored higher than 15 were allocated to the high levels group. This score is lower than what the authors outline as a cut-off for a significant level of traits, with only two participants in the current sample scoring 32 or higher. ASD is a spectrum condition, one in which each individual diagnosed may have symptoms or traits that are shared with and experienced by others as well as having some which are more unique to themselves. Therefore, results may not be generalisable to all individuals with ASD, due to the fact that each individual's experience with the condition is so unique. Also, the current sample consisted mainly of females, with only three males taking part in the study.

Considering that ASD is more common in males than females, (Carpenter, Happé \& Egerton, 2019), future research needs to use an equal number of male and female participants. This may even be useful to see whether there are any gender differences.

Although the study found no significant support for Hypothesis 1 relating level of autistic traits to the correct detection of fake news, there was support for the second hypothesis that individuals with high levels of ASD were less biased by the level of emotiveness in news articles, compared to those with low levels of ASD. Future studies using clinical samples may be able to investigate whether fake news detection is different in participants with a formal diagnosis. Further research is also needed to investigate whether those with ASD possess other skills that may relate to the cybersecurity sector which differ from neuro-typical participants. As suggested previously, the Cyber Neurodiversity Group suggest that adults diagnosed with ASD may have the skill sets needed to fill the current skill gap in the cybersecurity sector. Potential future empirical findings which support these suggestions could have an important influence in diversifying the sector and help the group in their current aims to utilise the skills of neuro-diverse individuals such as those with ASD.

\section{References}

Allcott, H., Gentzkow, M.: Social media and fake news in the 2016 election. Journal of Economic Perspectives, 31(2), 21136 (2017).

Bakir, V., McStay, A.: Fake news and the economy of emotions: Problems, causes, solution s. Digital Journalism, 6(2), 154175 (2018).

Baron-Cohen, S., Leslie, A. M., Frith, U.: Does the autistic child have a "theory of mind"? Cognition, 21(1), 37-46 (1985).

Baron-Cohen, S., Scott, F. J., Allison, C., Williams, J., Bolton, P., Matthews, F. E., Brayne, C.: Prevalence of autismspectrum conditions: UK school-based population studies. The British Journal of Psychiatry, 194(6), 500-509 (2009).

Baron-Cohen, S., Wheelwright, S., Skinner, R., Martin, J., Clubley, E.: The autism-spectrum quotient (AQ): Evidence from asperger syndrome/high-functioning autism, males and females, scientists and mathematicians. Journal of Autism and Developmental Disorders, 31(1), 5-17 (2001).

Bronstein, M. V., Pennycook, G., Bear, A., Rand, D. G., Cannon, T. D.: Belief in fake news is associated with delusionality, dogmatism, religious fundamentalism, and reduced analytic thinking. Journal of Applied Research in Memory and Cognition, 8(1), 108-117 (2019).

Brosnan, M., Chapman, E., Ashwin, C.: Adolescents with autism spectrum disorder show a circumspect reasoning bias rather than 'jumping-to-conclusions'. Journal of Autism and Developmental Disorders, 44(3), 513-520 (2014).

Cambridge Advanced Learner's Dictionary: 4th edition, https://dictionary.cambridge.org/dictionary/english/fake-news, last accessed 19/1/2020.

Carpenter, B., Happé, F., Egerton, J. (Eds.).: Girls and Autism: Educational, Family and Personal Perspectives. Routledge (2019).

Chesney, S., Liakata, M., Poesio, M., Purver, M.: Incongruent headlines: Yet another way to mislead your readers. In Proceedings of the 2017 EMNLP Workshop: Natural Language Processing meets Journalism (pp. 56-61) (2017). 
De Martino, B., Harrison, N. A., Knafo, S., Bird, G., Dolan, R. J.: Explaining enhanced logical consistency during decision making in autism. Journal of Neuroscience, 28, 10746-10750 (2008).

Goldstein, G., Johnson, C. R., Minshew, N. J.: Attentional processes in autism. Journal of Autism and Developmental Disorders, 31(4), 433-440 (2001).

Grabe, M. E., Zhou, S., Barnett, B.: Explicating sensationalism in television news: Content and the bells and whistles of form. Journal of Broadcasting \& Electronic Media, 45(4), 635-655 (2001).

Hayashi, M., Kato, M., Igarashi, K., Kashima, H.: Superior fluid intelligence in children with Asperger's disorder. Brain and Cognition, 66(3), 306-310 (2008).

Horne, B. D., Adali, S. This just in: fake news packs a lot in title, uses simpler, repetitive content in text body, more similar to satire than real news. In Eleventh International AAAI Conference on Web and Social Media (2017).

ICD-11 - Mortality and Morbidity Statistics, https://icd.who.int/browse11/1-m/en\#/http://id.who.int/icd/entity/437815624, last accessed 19/1/2020.

Jaeggi, S. M., Buschkuehl, M., Jonides, J., Perrig, W. J.: Improving fluid intelligence with training on working memory. Proceedings of the National Academy of Sciences, 105(19), 6829-6833 (2008).

Lazer, D. M., Baum, M. A., Benkler, Y., Berinsky, A. J., Greenhill, K. M., Menczer, F., Schudson, M.: The science of fake news. Science, 359(6380), 1094-1096 (2018).

Luke, L., Clare, I. C., Ring, H., Redley, M., Watson, P.: Decision-making difficulties experienced by adults with autism spectrum conditions. Autism, 16(6), 612-621 (2012).

Molek-Kozakowska, K.: Towards a pragma-linguistic framework for the study of sensationalism in news headlines. Discourse \& Communication, 7(2), 173-197 (2013).

Pennycook, G., Rand, D. G.: Lazy, not biased: Susceptibility to partisan fake news is better explained by lack of reasoning than by motivated reasoning. Cognition, 188, 39-50 (2019).

Pennycook, G., Cannon, T. D., Rand, D. G.: Prior exposure increases perceived accuracy of fake news. Journal of Experimental Psychology: General, September 24 (2018).

Shu, K., Sliva, A., Wang, S., Tang, J., Liu, H.: Fake news detection on social media: A data mining perspective. ACM SIGKDD Explorations Newsletter, 19(1), 22-36 (2017).

Smith, A.: The empathy imbalance hypothesis of autism: a theoretical approach to cognitive and emotional empathy in autistic development. The Psychological Record, 59(3), 489-510 (2009).

Stevenson, A. (Ed.): Oxford Dictionary of English (3rd edition), p.574. OUP: Oxford, UK (2010). 